\title{
Black carbon aerosol number and mass concentration measurements by picosecond short- range elastic backscatter lidar
}

Romain Ceolato ( $\nabla$ romain.ceolato@onera.fr)

Office National d'Études et de Recherches Aérospatiales

Andres Bedoya-Velasquez

Office National d'Études et de Recherches Aérospatiales

Frederic Fossard

Office National d'Études et de Recherches Aérospatiales

Vincent Mouysset

Office National d'Études et de Recherches Aérospatiales

Lucas Paulien

Office National d'Études et de Recherches Aérospatiales

Sidonie Lefebvre

Office National d'Études et de Recherches Aérospatiales

Claudio Mazzoleni

Michigan Technological University

Christopher Sorensen

Kansas State University

Matthew Berg

Kansas State University

Jerome Yon

University of Rouen

\section{Research Article}

Keywords: lidar, black, carbon, aerosol, range, time, resolution

Posted Date: August 19th, 2021

DOl: https://doi.org/10.21203/rs.3.rs-806433/v1

License: (c) (1) This work is licensed under a Creative Commons Attribution 4.0 International License.

Read Full License 
Version of Record: A version of this preprint was published at Scientific Reports on May 19th, 2022. See the published version at https://doi.org/10.1038/s41598-022-11954-7. 


\title{
Black carbon aerosol number and mass concentration measurements by picosecond short-range elastic backscatter lidar
}

\author{
Romain Ceolato ${ }^{1 *}$, Andrés E. Bedoya-Velásquez ${ }^{1}$, Frédéric Fossard ${ }^{2,3}$, Vincent \\ Mouysset ${ }^{1}$, Lucas Paulien ${ }^{1}$, Sidonie Lefebvre ${ }^{2}$, Claudio Mazzoleni ${ }^{6}$, Christopher \\ Sorensen ${ }^{4}$, Matthew J. Berg ${ }^{4}$, and Jérôme Yon ${ }^{7}$ \\ ${ }^{1}$ ONERA, The French Aerospace Lab, Toulouse University, FR 31055, France \\ ${ }^{2}$ ONERA, The French Aerospace Lab, Paris-Saclay University, FR 92322, Châtillon, France \\ ${ }^{3}$ CNRS, Laboratoire d'étude des microstructures, Paris-Saclay University, FR 92322, Châtillon, France \\ ${ }^{3}$ ONERA, The French Aerospace Lab, Palaiseau, France \\ ${ }^{4}$ Kansas State University, Department of Physics, 1228 N. 17 th St., Manhattan, KS 66506-2601, USA \\ ${ }^{6}$ Physics Department, Michigan Technological University, Houghton, Michigan, USA \\ ${ }^{7}$ Normandie University, UNIROUEN, INSA Rouen, CNRS, CORIA, Rouen 76000, France
}

\begin{abstract}
Black carbon aerosol emissions are recognized as contributors to global warming and air pollution. There remains, however, a lack of in-situ techniques to remotely quantify black carbon aerosol particles with high range and time resolution. This article presents for the first time, to our knowledge, a direct and contact-free remote measurement of black carbon aerosol number and mass concentration less than ten of meters from the emission source. This is done with a novel picosecond short-range elastic backscatter lidar (PSR-EBL). To address the complexity of retrieving lidar products at short measurement ranges, we apply a forward inversion method featuring radiometric lidar calibration. Our method is based on an extension of a well-established light-scattering model, the Rayleigh-Debye-Gans for Fractal-Aggregates (RDG-FA) theory, which computes an analytical expression of lidar parameters. These parameters are the backscattering cross-sections and the lidar ratio for black carbon fractal aggregates. Using a small-scale Jet A-1 kerosene pool fire, we demonstrate the ability of the technique to quantify the aerosol number and mass concentration with centimetre range-resolution and millisecond time-resolution.
\end{abstract}

\section{Introduction}

Black carbon (BC) particulate matter are light-absorbing aerosols produced from the incomplete combustion of hydrocarbon fuel and biomass ${ }^{1}$. These particles consist of fractal aggregates of ultra-fine primary soot monomers, which are substantial climate-forcing agents due to their strong absorption of visible solar radiation in the atmosphere ${ }^{2-4}$. Black carbon emissions also influence the cloud formation processes which can impact regional circulation and rainfall patterns ${ }^{5}$. Moreover, these particles pose a threat to human health as they are considered a carcinogen and source of respiratory disease due to their nanometer size ${ }^{6}$; they also constitute a negative influence on urban air quality ${ }^{7}$. In other contexts, BC aerosols emitted by aircraft engines (also known as non-volatile particle matter) are potential ice nuclei and may induce cirrus clouds ${ }^{8-10}$. Through this mechanism, BC aerosols may present climate forcing that could be comparable to the cumulative impacts of $\mathrm{CO}_{2}$ emissions ${ }^{11}$. Yet, substantial uncertainties remain surrounding the net climate forcing of $\mathrm{BC}$ aerosols because of the large variety of substances encompassing freshly emitted as well as aged soot. Thus, the quantification of BC aerosol emissions, meaning aggregate-particle number $n_{\mathrm{o}}$ and mass concentration $m_{\mathrm{o}}$, is essential to advance our understanding of their role in both global warming and environmental health $^{12,13}$.

A variety of techniques are available to characterize BC-aerosols such as filter-based absorption photometer ${ }^{14}$, photoacoustic measurements ${ }^{15}$, photothermal interferometry ${ }^{16}$, aethalometry ${ }^{17}$, or light-scattering principles ${ }^{18}$. For example, the Soot Photometer (SP2) instrument employs laser-induced incandescence and light scattering ${ }^{19}$ as an in-situ technique to measure the size and volume fraction of BC particles ${ }^{20}$. The Scanning Mobility Particle Sizer (SMPS) can determine particle size and Soot Particle Aerosol Mass Spectrometry (SP-AMS) can provide on-line analysis BC-particle chemical compounds ${ }^{21}$. For all of these instruments and techniques, the measurements are local in nature, i.e., they do not provide at-a-distance range-dependent measurements and several of them are not widely used due to their cost and complex design ${ }^{22}$. Elastic backscatter lidar (EBL), however, is an active remote-sensing technique with the ability to characterize aerosols in a contact-free manner ${ }^{23-26}$. Such 
lidar operates by measuring laser light elastically scattered in the backward direction from an ensemble of particles. Until recently, EBL instruments mostly employ nanosecond pulsed laser sources to probe the atmosphere with several-meter range resolution and several seconds to minutes time-resolution ${ }^{27,28}$. Generally, EBL instruments are rarely used for short-range applications due to an incomplete overlap between the outgoing laser beam and the receiver field-of-view. Environmental and air quality applications ${ }^{29-35}$ have recently raised a need for aerosol characterization close to the emission source, which is driving a decreasing to the minimal measurement-range in EBL technology.

Here, we report on a novel in-situ remote sensing EBL technique to quantify BC number and mass concentration. We demonstrate the feasibility of remote measurement of BC aerosols within the first tens of meters along the line of sight from the emission source with our instrument using a picosecond laser. These measurements feature a high degree of range and time-resolution. To our knowledge, there exist no published attempts to accurately measure concentrations of ultrafine particulate matter, such as BC, via backscatter measurements with such high resolution close to the emission source. The following will present the EBL instrument and a proof-of-principle measurement involving a Jet A1 kerosene pool-fire as a source for a BC aerosol. A lidar inversion method will be described that features an analytical model for lidar-relevant parameters (i.e. backscattering, and lidar ratio) based on the Rayleigh-Debye-Gans for Fractal-Aggregates (RDG-FA) theory. The results provided by our work should meet the growing need for BC particle measurements and could be assimilated into atmospheric transport models ${ }^{36-38}$, combustion-related issues for indoor ${ }^{39}$ or outdoor fires ${ }^{40}$, and health problems ${ }^{41,42}$.

\section{Results}

\section{Principle of operation}

The Picosecond Short-Range Elastic Backscatter Lidar (PSR-EBL) is an active remote-sensing technique designed to measure the number and mass concentration profiles of ultrafine particulate matter in a range-resolved manner. In this study, the ultrafine matter is a BC aerosol. The principle of operation is illustrated in Fig. 1 and is described in further detail in the Methods section. Here, a series of picosecond pulses are emitted from the lidar transmitter, illuminating a column of aerosol particles in the $\hat{\mathbf{q}}^{\text {inc }}$ direction. When a pulse arrives at a particle located at $\mathbf{r}$ as shown in the inset in Fig. 1, it may be partly absorbed and will scatter in all directions $\hat{\mathbf{q}}$. The return-signal consists of the portion of light backscattered to the lidar receiver's area $A$, which defines the received solid angle $\Delta \Omega$ centered on the backscattering direction $-\hat{\mathbf{q}}^{\text {inc }}$. The position of the particle relative to the lidar, $\mathbf{r}$, depends on the range $r$ as $\mathbf{r}=r \hat{\mathbf{q}}^{\text {inc }}$ as shown in Fig. 1 . Then, the backscattered specific intensity $\widetilde{\mathbf{I}}^{\text {bac }}\left(\mathbf{r},-\hat{\mathbf{q}}^{\text {inc }}, t\right)$ can be directly derived from the Radiative Transfer Equation (RTE) ${ }^{26}$ as,

$$
\widetilde{\mathbf{I}}^{\mathrm{bac}}\left(\mathbf{r},-\hat{\mathbf{q}}^{\mathrm{inc}}, t\right)=\frac{c \tau}{2} \mathbf{U}(\mathbf{r}, t) \cdot \widetilde{\mathbf{I}}^{\mathrm{inc}}\left(\mathbf{r}, \hat{\mathbf{q}}^{\mathrm{inc}}\right)
$$

where $c$ is the speed of light, $\tau$ is the laser pulse duration, $\widetilde{\mathbf{I}}^{\text {inc }}\left(\mathbf{r}, \hat{\mathbf{q}}^{\text {inc }}\right)$ is the incident specific intensity, and $\mathbf{U}(\mathbf{r}, t)$ is the attenuated backscattering Stokes matrix. For the polarization insensitive measurements considered here, Eq. (1) can be simplified ${ }^{26}$ by a scalar version of $\mathbf{U}$ as

$$
U(\mathbf{r}, t)=n_{\mathrm{o}}(\mathbf{r}, t)\left\langle Z_{11}\left(\hat{\mathbf{q}}^{\text {inc }},-\hat{\mathbf{q}}^{\text {inc }}\right)\right\rangle \exp \left[-2 n_{\mathrm{o}}(\mathbf{r}, t) \int_{0}^{r}\left\langle C^{\text {ext }}\left(\mathbf{r}^{\prime}\right)\right\rangle \mathrm{d} \mathbf{r}^{\prime}\right]
$$

where $n_{\mathrm{o}}(\mathbf{r}, t)$ is the range and time dependent number concentration of particles, $\langle$.$\rangle is the ensemble-averaged operator,$ $Z_{11}\left(\hat{\mathbf{q}}^{\text {inc }},-\hat{\mathbf{q}}^{\text {inc }}\right)$ is the first element of the Stokes phase matrix with units of area per solid angle, and $C^{\text {ext }}$ is the extinction cross-section per particle with units of area.

The lidar instrument in this work is a forward-looking PSR-EBL system, named Colibri, which employs a high repetition rate laser of short pulses, permitting backscatter measurements with a millisecond time and centimeter range-resolution using the time-of-flight principle for distance determination. This is in contrast to conventional lidar systems intended for atmospheric studies. The instrument operated for several hours on February $20^{\text {th }}, 2021$ at an outdoor facility at ONERA in Occitanie, France. $\mathrm{BC}$ aerosols are generated from the combustion of a pool of aviation fuel (Jet A-1 kerosene), which is a sulfur-containing complex mixture of various hydrocarbons and alkanes. The small-scale pool fire generates plumes of soot at a range of $10 \mathrm{~m}$ from the Colibri system. The efficiency of the pool-fire depends on several parameters including environmental conditions (wind, temperature, ambient pressure), and the $\mathrm{BC} n_{\mathrm{o}}$ and $m_{\mathrm{o}}$ are continuously characterized in the experiment using an optical particle counter (Palas, Fidas 200). A first proof-of-principle of PSR-EBL measurements is shown at the bottom of Fig. 1. Here, the range-corrected backscatter signals, which are directly related to the amount of BC aerosol, i.e., $n_{\mathrm{o}}$, are displayed for 4.5 seconds, at a distance of nine meters, and a height of 1.20 meters above the pool-fire. A methodology will be described below to retrieve the range and time dependent profiles of $\mathrm{BC} n_{\mathrm{o}}$ and $m_{\mathrm{o}}$ from the return signals. 


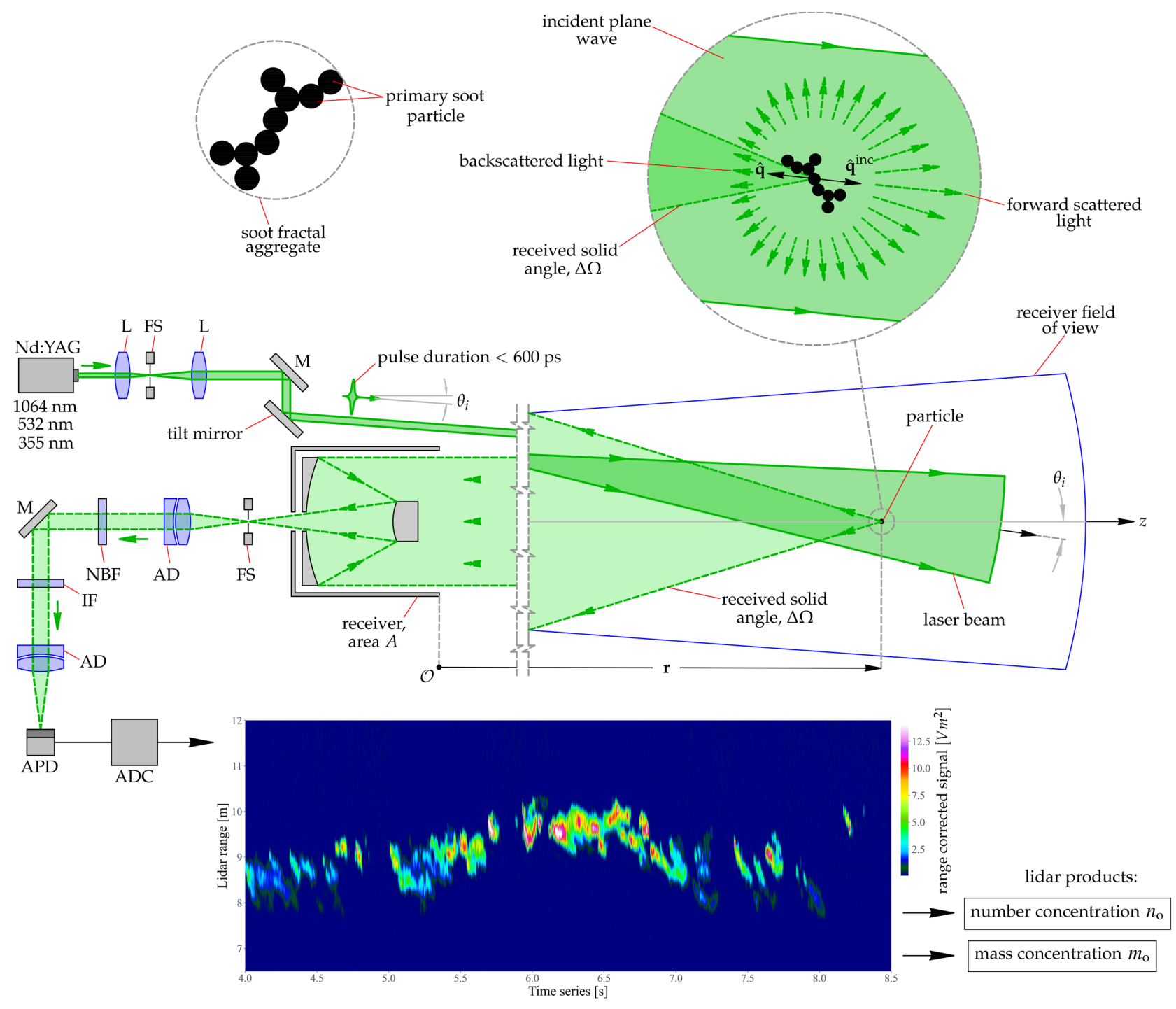

Figure 1. Principle of operation of the Picosecond Short-Range Elastic Backscatter Lidar (PSR-EBL) technique, intended to measure $\mathrm{BC}$ aerosol number and mass concentration, $n_{\mathrm{o}}$ and $m_{\mathrm{o}}$, respectively. A picosecond laser pulse is emitted from the lidar transmitter to illuminate a column of $\mathrm{BC}$ aerosols in the direction $\hat{\mathbf{q}}^{\text {inc }}$. When a pulse arrives at a particle (shown inset) at a range $r$, it may be partly absorbed and will scatter in all directions $\hat{\mathbf{q}}$. The lidar return signal is directly related to the light backscattered by the particle to the receiver's area $A$, which defines the received solid angle $\Delta \Omega$. An example measurement of the return signal is shown at the bottom for a small-scale kerosene pool-fire at a range of $9 \mathrm{~m}$ from the instrument. Further description of the Colibri lidar is given in the Methods section. 


\section{Microphysics of the BC particles}

Using Scanning Transmission Electron Microscopy with a High Angle Annular Dark Field (STEM/HAADF) feature, BC aggregates collected on copper TEM grids at 1.2 meters above the Jet-A1 pool-fire are characterized as shown in Fig. 2. One can see that the BC particles consist of clusters of carbonaceous primary particles, or monomers, with a high fraction of graphite-like $\mathrm{sp}^{2}$-bonded carbon atoms. In the STEM/HAADF mode, electrons from a nanometric probe are elastically scattered by the particle nuclei and collected by an annular detector to form the image contrast seen. Incoherent scattering of this kind provides simpler analysis of the image contrast by minimizing the dynamic effects that hamper conventional bright-field images. Consequently, the contrast values depend only on the number and type of atoms scattering the electrons. As such, the intensity collected by the image sensor can be directly linked to the thickness of the sample, assuming that the composition of the material is homogeneous ${ }^{43}$.
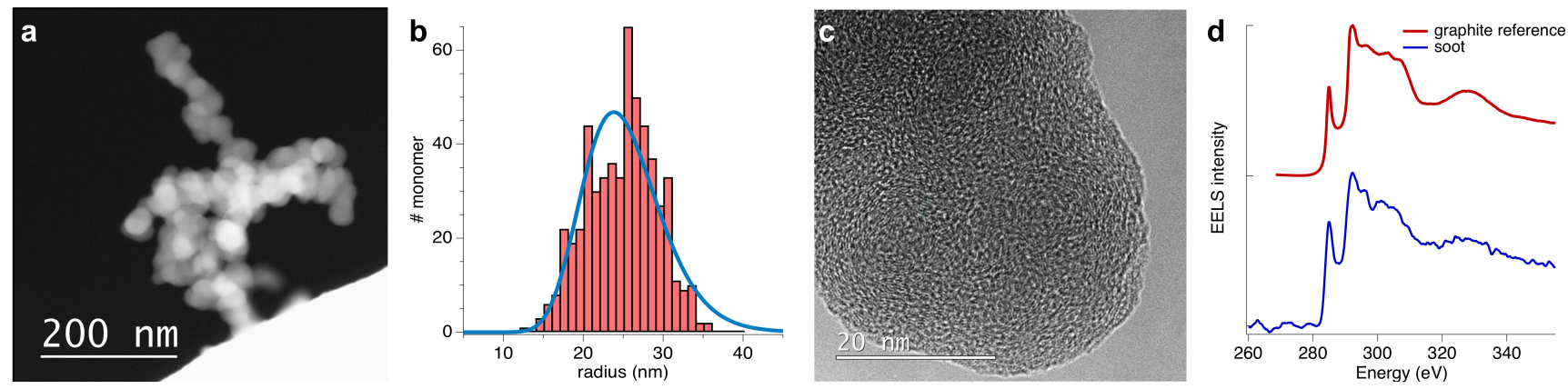

Figure 2. Microphysical properties of BC particles from a Jet-A1 pool-fire. In (a) is an STEM/HAADF image of a typical BC aggregate, while (b) shows the size distribution, in radius, of the monomers (red bars) and its lognormal fit (blue). In (c), a HRTEM image of a monomer is shown illustrating an onion-like structure and (d) presents the C-K edge EEL spectra of a monomer in blue and for graphite in red as a reference.

Figure 2(a) shows a STEM/HAADF image of typical BC aggregate containing approximately 90-100 monomers. The monomers present a nearly spherical shape with a diameter smaller than $100 \mathrm{~nm}^{3}$ and form a fractal-like aggregate with a typical fractal dimension ${ }^{44}$ of $D_{\mathrm{f}}=1.8$ and up to hundreds of nanometers in size. The monomer size distribution, shown in Fig. 2(b) in terms of monomer radius, is obtained by measuring at least 550 monomers from 10 different aggregates (see examples in SI) and is fitted with a lognormal distribution. The mean radius is $R_{\mathrm{m}}=23.8 \pm 0.4 \mathrm{~nm}$, which will be used later in the the LIDAR inversion model. Using this distribution in combination with the aggregate size deduced from the contrast in the image and its projected area, the aggregate volume can be deduced. That information then estimates the average number of monomers and the surface area of the aggregate assuming that necking between the monomers is negligible. Further characterization of the monomers is shown by the high-resolution TEM (HRTEM) analysis in Fig. 2(c) where the onion-like structure with randomly-orientated fringes indicates a microstructure typical of carbon black with its turbostratic stacking ${ }^{45-47}$.

Electron Energy Loss Spectroscopy (EELS) is also performed at the carbon K-edge on several monomers. A typical EEL spectrum of a black carbon soot is shown in blue in Fig. 2(d). The shape of the edge presents several features which are well-known and related to $\mathrm{sp}^{2}$-hybridized carbon. Indeed, the first peak at $285 \mathrm{eV}$ corresponds to the transition between the carbon 1s state and the first lowest unoccupied molecular orbital, which in this case is a $\pi^{*}$ orbital. The second peak is related to the higher anti-bonding orbital $\sigma^{*}$ of carbon. One tried to quantify the amount of $\mathrm{sp}^{2}$-hybridized carbon by EELS by studying the peak area ratio $\pi^{*}$ to $\pi^{*}+\sigma^{* 48}$. We used this criterion to estimate the amount of graphitic (aromatic cycles) versus amorphous carbon (C-H bonds) and it reveals a strong anisotropy of the graphitic structure combined with the spherical morphology of the monomers ${ }^{49,50}$. Thus, we assume hereby that the soot can be assimilated to pure $\mathrm{sp}^{2}$-hybridized carbon by directly comparing to an highly oriented pyrolytic graphite reference. Therefore, the optical properties and especially the dielectric function of $\mathrm{BC}$ can be used for LIDAR data analysis.

\section{Number and mass concentration}

The lidar return signals, which are related to Eq. (2), must be inverted to retrieve an estimate of the BC aerosol number and mass concentration, $n_{\mathrm{o}}$ and $m_{\mathrm{o}}$, respectively. While the details of this inversion are given in the Methods section, it involves lidar products obtained at three levels:

(i) The first-level products are the attenuated backscatter profiles $\mathrm{U}(r, t)$ of Eq. (2), which are the range corrected lidar signals resulting from the application of a radiometric calibration ${ }^{51}$. The lidar signals are pre-processed to increase the 
signal-to-noise ratio. Here, this pre-processing consists of a dark current correction (DC), a background correction (BG), and denoising by means of a low pass filter that preserves the range resolution of the original signal ${ }^{52}$.

(ii) The second-level products are backscatter profiles $\beta(r, t)$ obtained from a forward lidar-inversion method applied directly to the $\mathrm{U}(r, t)$ signals. The inversion uses a light-scattering model that accounts for the fractal morphology of BC aerosols and is an essential element in determining accurate backscatter profiles from PST-EBL measurements. Here, the lidar ratio is calculated using the Rayleigh-Debye-Gans for Fractal Aggregates (RDG-FA) theory and the microphysical parameters provided by the STEM/HAADF analysis.

(iii) Lastly, third-level products are the $\mathrm{BC}$ aerosols number and mass concentration range and time-dependent profiles $n_{\mathrm{o}}(r, t)$ and $m_{\mathrm{o}}(r, t)$. These are calculated using, respectively, the differential backscattering cross-section $\mathrm{d} C^{\text {bac }}$ and mass-specific backscattering efficiency $\sigma^{\text {bac }}$ for BC fractal aggregates via RDG-FA theory.

Examples of the third-level lidar products $n_{\mathrm{o}}(t, r)$ and $m_{\mathrm{o}}(t, r)$ are presented in Fig. 3. The measurements display two plumes of $\mathrm{BC}$ emitted from the pool-fire at approximately $9 \mathrm{~m}$ from the lidar instrument between $0-5 \mathrm{~s}$ and 20-25 s. These results demonstrate the ability of the PSR-EBL to perform contact-free measurements at a range-resolution of $5 \mathrm{~cm}$ and a time-resolution of $4 \mathrm{~ms}$, which is revealed by the insets of the later-time plume in Fig. 3. For the plume displayed in the inset specifically, the spatio-temporal mean of the number and mass concentration is found to be, respectively, $\left\langle n_{\mathrm{o}}\right\rangle=7395$ particles $/ \mathrm{cm}^{3}$ with standard deviation up to $\sigma_{n}=4900$ particles $/ \mathrm{cm}^{3}$ and $\left\langle m_{\mathrm{o}}\right\rangle=0.069 \mathrm{mg} / \mathrm{m}^{3}$ with a standard deviation of $\sigma_{m}=0.05 \mathrm{mg} / \mathrm{m}^{3}$. The standard deviation refers to the range and time variability of these products within this plume.

(a) number concentration $n_{\mathrm{o}}(r, t)$

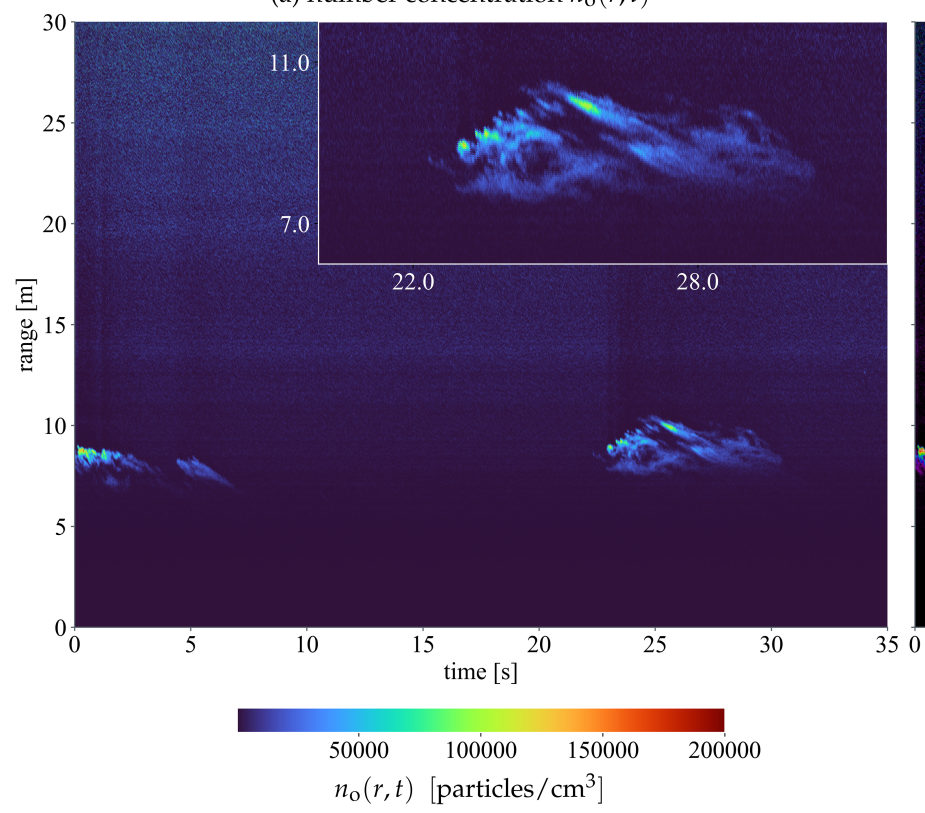

(b) mass concentration $m_{\mathrm{o}}(r, t)$

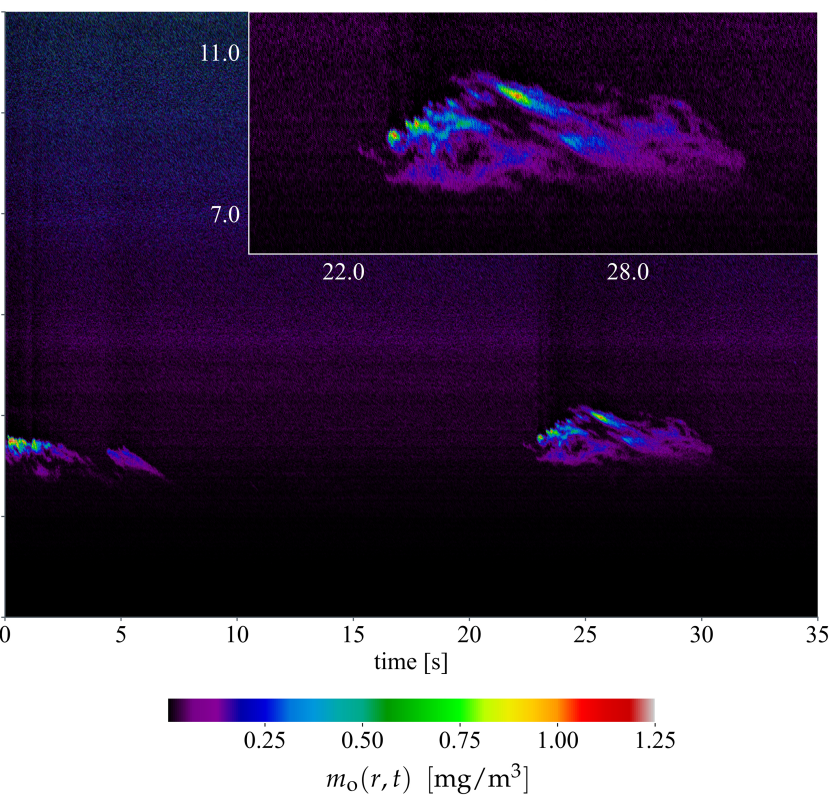

Figure 3. Range and time-resolved number $n_{\mathrm{o}}(r, t)$ and mass $m_{\mathrm{o}}(r, t)$ concentration profiles from the Colibri PSR-EBL measurements of BC aerosols emitted by a small-scale Jet A-1 pool-fire. To highlight the resolution obtained, the inset images show a magnified view of the plume occurring around 25 seconds.

With the understanding that a comparison of different sensors is complicated by differences in measurement techniques, the level of agreement between BC mass concentrations measured by a local sensor (Fidas 2000) and the Colibri PSR-EBL is examined. The time-averaged number and mass concentration as a function of range are computed in the Supplementary information. The mean number concentration measured by the Fidas 2000 instrument is $\left\langle n_{\mathrm{o}}\right\rangle=8020 \pm 1842 \mathrm{particles} / \mathrm{cm}^{3}$ and the mean mass concentration was approximately $\left\langle m_{\mathrm{o}}\right\rangle=0.02 \pm 0.02 \mathrm{mg} / \mathrm{m}^{3}$. Thus, a good agreement is found between the FIDAS and PSR-EBL measurements. The difference in standard deviation between the Fidas and Colibri lidar measurements is within the margins of errors of the different measurement techniques involved. It could be due to the nature of the instruments and the different temporal resolution between them, i.e. $2 \mathrm{~min} / \mathrm{sample}$ for FIDAS and $4 \mathrm{~ms} / \mathrm{sample}$ for the PSR-EBL lidar. The salient point is that the comparison between local and remote sensors does indeed show good agreement. 


\section{Discussion}

While BC particulate matter are typically characterized by local sensors, there is a growing need for short-range remotesensing techniques ${ }^{28,53}$. Indeed, most sensors currently available do not provide range-dependent profiles as they rely on arrays of spatially distributed and time-integrating samplers to collect particles for analysis. A prominent advantage of active remote-sensing techniques, such as the PSR-EBL lidar described here, is that they do not require spatial interpolation or aerosol-dispersion models as they directly provide the range and time-resolved measurements.

Our findings have important implications that overcome several limitations of conventional EBL techniques. One such limitation relates to lidar range and time resolution. Results from the Colibri PSR-EBL instrument can be used to estimate the $\mathrm{BC}$ aerosol number and mass concentration with centimeter range-resolution and millisecond time-resolution, by virtue of the picosecond laser and fast return-signal sensor. A second limitation concerns the capability of measuring aerosol concentration in the short range. Most EBL instruments are "blind" in the short-range due to an incomplete overlap between the emitted laser beam and receiver field of view. Here, however, we demonstrate that this limitation can be relived and concentration profiles obtained at ranges of 8-10 meters in the outdoor environment through a bi-static bi-axial lidar configuration. A third limitation concerns the retrieval methods needed to invert the return signals to retrieve the aerosol characteristics of interest. Common EBL inverse-methods rely on assumptions such as an aerosol-free zone. While here the inverse method requires a prior radiometric calibration of the system, it does enable the accurate measurements in any environmental condition, without the need for a reference or a clean molecular zone in the atmosphere, i.e. molecular normalization ${ }^{54}$. And lastly, most inversion methods in remote-sensing rely on spherical or spheroidal proxies for the aerosol particle in calculating the aerosol lidar-relevant properties, i.e., the lidar ratio and backscattering cross-sections. Through use of the RDG-FA theory, these quantities are pre-calculated and account for the fractal morphology of the $\mathrm{BC}$ particles, including such physically relevant parameters as monomer size and aggregate fractal-dimension.

The results presented here demonstrate the potential of the PSR-EBL to measure the range and time-resolved number and mass concentrations of $\mathrm{BC}$ emissions. While the high range and time-resolution and short-range capabilities provide a new approach in lidar for such particle measurements, further work is necessary to evaluate the full capabilities of the technique. Future studies could better assess the level of agreement between the PSR-EBL and other well-established techniques such as SMPS or SP-AMS. Nevertheless, the relative error on the retrieved concentrations was estimated to be less than $16 \%$ for number concentration and 25\% for mass concentration by an error propagation analysis (See Supplementary information for details), which is consistent with other similar studies ${ }^{55,56}$. Future developments in PSR-EBL technique could provide improved insight for BC-aerosol emission studies, especially given that our work is subject to limitations, including the need for detailed microscopy of representative BC particles. As another improvement, a multi-wavelength picosecond laser could be used to infer more detail regarding the optical properties of $\mathrm{BC}$-aerosol particles, including for example, the monomer particle-size, the presence of aggregate aging, and even distinguishing black- from brown-carbon aggregates.

\section{Methods}

\section{Short-range micro-lidar instrument}

The Colibri instrument is a forward-looking picosecond short-range elastic backscatter lidar (PSR-EBL) with a bi-static, multi-axial architecture. The system was developed by ONERA, The French Aerospace Lab, for remote measurements of aerosols with high range and temporal resolution ${ }^{26,51}$. Compared to other lidar systems for atmospheric studies, Colibri is lightweight, compact, and suitable for a mobile platform. The transmitter unit is composed of a compact air-cooled Nd:YAG laser that emits 600 ps pulses with a pulse energy of $25 \mu \mathrm{J}$, wavelength of $\lambda=532.8 \mathrm{~nm}$, repetition rate of $1 \mathrm{kHz}$, and a beam divergence of $0.5 \mathrm{mrad}$. As shown in Fig. 1, the linear-polarized beam is reflected and directed towards the aerosols of interest using a tilt mirror. A bi-static architecture is preferred over a mono-static configuration to prevent pulses from causing internal reflections that could saturate the sensor and thus reduce the minimum range of measurement. The bi-static angle, i.e. the angle $\theta_{i}$ subtended between the transmitter and receiver units, is an essential feature for short-range measurements as it enables full control of the overlap function. The receiver unit employs a Cassegrain telescope with a $90 \mathrm{~mm}$ effective diameter and $500 \mathrm{~mm}$ focal length. Light collected by the telescope is focused on the sensor by a pair of achromatic doublet (AD) lenses, with a neutral-band filter (NBF) and interference filter (IF) to control light levels. For short-range measurements, the optical elements following the telescope is placed on a translation stage, allowing adjustment of the lidar focal plane. This feature is essential for short-range lidar measurements as it resolves focusing problems encountered for short ranges. For the measurements reported here, the focal plane position is set to maximize the collected signal magnitude at a range of approximately $10 \mathrm{~m}$. The sensing unit is a high-bandwidth silicon avalanche photodiode (APD) and its analog signal is passed to a high bandwidth single-channel Digital Signal Processing (DSP) to digitize the signal. 


\section{Inversion method}

The elastic lidar equation can be derived from the Radiative Transfer Equation (RTE) ${ }^{26}$, which itself can be derived from first principles, i.e., from the Maxwell equations, as shown by Mishchenko ${ }^{57}$. The RTE usually assumes that the incident light is scattered only once, i.e., multiple-scattering events are ignored, and thus, can be analyzed as a link-budget for the backscattered power $\mathrm{P}(r)$ as a function of range $r$ as:

$$
\mathrm{P}(r)=\mathrm{K}_{\mathrm{o}} \mathrm{O}(r) \mathrm{U}(r) r^{-2}
$$

where $\mathrm{K}_{\mathrm{o}}$ is the radiometric lidar constant, $\mathrm{O}(r)$ is the range-dependent overlap function accounting for the partial overlap between the lidar field of view and the laser beam, and $\mathrm{U}(r)$ is the attenuated backscattering function defined as:

$$
\mathrm{U}(r)=\beta(r) \exp \left(-2 \int_{0}^{r} \alpha\left(r^{\prime}\right) \mathrm{d} r^{\prime}\right)
$$

with units of inverse distance time inverse solid angle. In Eq. (4), $\alpha=\alpha_{\mathrm{bc}}+\alpha_{\mathrm{mol}}$ and $\beta=\beta_{\mathrm{bc}}+\beta_{\mathrm{mol}}$ which represent the total extinction and backscattering coefficients as sums of the BC aerosol (bc) and the molecular components (mol), i.e., that due to the gas molecules in the atmosphere. The ill-posed nature of the lidar inversion problem requires the extinction-to-backscattering ratio, or lidar ratio (LR) to be assumed ${ }^{58-60}$. This ratio is defined for BC particles and molecular components, respectively, as $\mathrm{LR}_{\mathrm{bc}}=\alpha_{\mathrm{bc}} / \beta_{\mathrm{bc}}$ and $\mathrm{LR}_{\mathrm{mol}}=\alpha_{\mathrm{mol}} / \beta_{\mathrm{mol}}$.

We now describe a method to retrieve the BC backscattering coefficient directly from calibrated radiometric micro-lidar measurements. Equation (4) is converted to a form ${ }^{61}$ involving a single unknown, $\mathrm{LR}_{\mathrm{bc}}$. With $\mathrm{LR}_{\text {mol }}$ regarded as known ${ }^{62}$, this form is obtained by splitting the exponential term into two parts so that only the total backscattering coefficient appears:

$$
\mathrm{U}(r)=\beta(r) \exp \left[-2 \int_{0}^{r} \mathrm{LR}_{\mathrm{bc}} \beta\left(r^{\prime}\right) \mathrm{d} r^{\prime}\right] \exp \left[-2 \int_{0}^{r}\left(\mathrm{LR}_{\mathrm{mol}}-\mathrm{LR}_{\mathrm{bc}}\right) \beta_{\mathrm{mol}}\left(r^{\prime}\right) \mathrm{d} r^{\prime}\right] .
$$

Because $\beta_{\text {mol }}$ is commonly predicted from air density profiles, Eq. (5) can be further simplified via

$$
\mathrm{V}(r)=\beta(r) \exp \left[-2 \int_{0}^{r} \operatorname{LR}_{\mathrm{bc}} \beta\left(r^{\prime}\right) \mathrm{d} r^{\prime}\right]
$$

with units of inverse distance time inverse solid angle. In Eq. (6), V(r) is a modified attenuated backscattering function, which is related to Eq. (5) as

$$
\mathrm{V}(r)=\mathrm{U}(r) \exp \left[2 \int_{0}^{r}\left(\mathrm{LR}_{\mathrm{mol}}-\mathrm{LR}_{\mathrm{bc}}\right) \beta_{\mathrm{mol}}\left(r^{\prime}\right) \mathrm{d} r^{\prime}\right] .
$$

Equation (6) now contains a single unknown, $\beta$, which yields an analytical solution ${ }^{51}$ as:

$$
\beta(r)=\beta_{\mathrm{bc}}(r)+\beta_{\mathrm{mol}}(r)=\frac{\mathrm{V}(r)}{1-2 \mathrm{LR}_{\mathrm{bc}} \int_{0}^{r} \mathrm{~V}\left(r^{\prime}\right) \mathrm{d} r^{\prime}} .
$$

Equation. (8), however, requires fine-scale evaluation of the exponential term in Eq. (7), which can become a source of growing numerical errors. The resolution adopted here is to simultaneously evaluate this term along with Eq. (6). Following Ceolato $^{51}$, two admittance quantities, $T(r)$ and $W(r)$, are introduced

$$
\mathrm{T}(r)=\frac{\mathrm{V}(r)}{\beta(r)}=\exp \left[-2 \int_{0}^{r} \mathrm{LR}_{\mathrm{bc}} \beta\left(r^{\prime}\right) \mathrm{d} r^{\prime}\right] \quad \text { and } \quad \mathrm{W}(r)=\frac{\mathrm{V}(r)}{\mathrm{U}(r)}=\exp \left[-2 \int_{0}^{r}\left(\mathrm{LR}_{\mathrm{bc}}-\mathrm{LR}_{\mathrm{mol}}\right) \beta_{\mathrm{mol}}\left(r^{\prime}\right) \mathrm{dr}^{\prime}\right] .
$$

These can now be seen as solutions to the system of coupled first-order partial differential equations

$$
\left\{\begin{aligned}
\partial_{r} \mathrm{~W}(r) & =-2\left(\mathrm{LR}_{\mathrm{mol}}-\mathrm{LR}_{\mathrm{bc}}\right) \beta_{\mathrm{mol}}(r) \mathrm{W}(r), \\
\partial_{r} \mathrm{~T}(r) & =-2 \mathrm{LR}_{\mathrm{bc}} \mathrm{U}(r) \mathrm{W}(r), \\
\mathrm{T}(0) & =\mathrm{W}(0)=1
\end{aligned}\right.
$$

The system in Eq. (10) is solved and $\beta_{\mathrm{bc}}$ is given by

$$
\beta_{\mathrm{bc}}(r)=\frac{\mathrm{U}(r) \mathrm{W}(r)}{\mathrm{T}(r)}-\beta_{\mathrm{mol}}(r)
$$


Next, $\beta_{\mathrm{bc}}(r)$ is used to calculate $n_{\mathrm{o}}(r)$ and $m_{\mathrm{o}}(r)$, respectively, as:

$$
\beta_{\mathrm{bc}}(r)=\int_{R_{\min }}^{R_{\max }} n_{\mathrm{o}}\left(r, R_{g}\right) \mathrm{d} C^{\mathrm{bac}}\left(r, R_{g}\right) \mathrm{d} R_{g}
$$

where $n_{\mathrm{o}}\left(r, R_{g}\right)$ is the particle number concentration per unit volume for an isotropic scattering medium formed by an ensemble of randomly oriented $\mathrm{BC}$ aggregates with radius of gyration $R_{\mathrm{g}}$. In Eq. (12), $R_{\min }$ and $R_{\max }$ are the minimum and maximum radii of gyration, and $\mathrm{d} C^{\mathrm{bac}}$ is the differential backscattering cross-section of a $\mathrm{BC}$ aggregate, which is defined as:

$$
\mathrm{d} C^{\mathrm{bac}}\left(r, R_{g}\right)=\left.\frac{d \sigma^{\mathrm{sca}}\left(r, R_{g}\right)}{d \Omega}\right|_{\theta=\pi}
$$

and has units of surface time inverse solid angle. For a given mass specific backscattering efficiency $\sigma^{\text {bac }}$, the mass concentration of $\mathrm{BC}$ particulate matter is ${ }^{28,63}$ :

$$
m_{\mathrm{o}}(r, R)=\frac{\beta_{\mathrm{bc}}(r)}{\sigma^{\mathrm{bac}}}
$$

with units of mass time inverse volume $\mathrm{mg}^{2} / \mathrm{m}^{3}$. Note that $\sigma^{\text {bac }}$ is defined from the mass specific extinction coefficient $\sigma^{\text {ext }}$ and the lidar ratio for $\mathrm{BC}$ as:

$$
\sigma^{\mathrm{bac}}=\frac{\sigma^{\mathrm{ext}}}{L R}
$$

with units of surface time inverse solid angle and inverse mass $\left[\mathrm{m}^{2} /(\mathrm{sr} \cdot \mathrm{mg})\right]$.

\section{Rayleigh-Debye-Gans for Fractal Aggregates theory}

Several accurate electromagnetic scattering methods are available to simulate the radiative properties of BC aggregates in a numerically exact manner. Perhaps the most flexible is the Discrete Dipole Approximation ${ }^{64}$ (DDA). Yet, it remains difficult to implement such methods given the significant computational time required when they are used for lidar inversion. Thus, approximate models of the radiative properties remain justified. Here, the approximation pursued is the Rayleigh-Debye-Gans for Fractal Aggregates (RDG-FA) theory, which is shown to be accurate to model light-scattering of fractal aggregates ${ }^{65}$, including lidar-relevant quantities ${ }^{66}$, and in controlled laboratory experiments ${ }^{67}$. Using the RDG-FA theory, the backscattering and extinction cross-sections can be simply and analytically derived, and then, used for the lidar inversion.

Because an aggregate's monomers are small compared to $\lambda$, one can assume that the wave phase shift across a monomer is negligible; this is one aspect of the RDG-FA theory. Doing so is equivalent to assuming that the electromagnetic field within a monomer is uniform, which is valid for spherical monomers when $x_{\mathrm{m}}|m-1| \ll 1$ where $m=n+i \kappa$ is the complex refractive index and $x_{\mathrm{m}}=2 \pi R_{\mathrm{m}} / \lambda$ is the monomer size-parameter. In this case, the monomer will scatter in the so-called Rayleigh limit. The other assumption in RDG-FA theory is that across an aggregate, the monomers scatter the incident light independent of each other, i.e., monomer-monomer multiple scattering is neglected. We note that these assumptions have limitations and a summary is given at the end of this section. The RDG-FA theory then postulates that an aggregate's absorption cross section is the sum of the cross sections for each monomer. While the condition $x_{\mathrm{m}}|m-1| \ll 1$ may be justified at the monomer level, it is not at the aggregate level due to the increased size resulting from the assemblage of many monomers. Thus, some care is needed to approximate the differential scattering cross section since there can be a significant phase shift across the aggregate.

Provided that the lidar beam is vertically polarized and the received scattered light is also vertically polarized, the differential scattering cross section $\mathrm{d} C_{\mathrm{bc}}^{\text {sca,vv }}$ of a $\mathrm{BC}$ aggregate is proportional to the squared number of monomers $N_{\mathrm{m}}$, the scattering cross section of an individual monomer $\mathrm{d} C_{\mathrm{m}}^{\text {sca,vv }}$, and a function $f$, called structure factor, that accounts for the fractal structure of the aggregate. The structure factor depends on $R_{\mathrm{g}}$, the scattering angle $\theta$, and the aggregate's fractal dimension $D_{\mathrm{f}}$, thus

$$
\mathrm{d} C^{\mathrm{sca}, \mathrm{vv}}=N_{\mathrm{m}}^{2} \mathrm{~d} C_{\mathrm{m}}^{\mathrm{sca}, \mathrm{vv}} f\left(R_{\mathrm{g}}, \theta, D_{\mathrm{f}}\right) .
$$

We note that different expressions for $f$ are reported in the literature ${ }^{44,68}$. Each formulation involves the scattering wave vector $q(\theta, \lambda)=(4 \pi / \lambda) \sin (\theta / 2)$. Here, we use that formulated by Dobbins and Megaridis ${ }^{69}$ due to its simplicity and because it is known to be accurate at $\lambda=532 \mathrm{~nm}$ even when internal monomer-monomer multiple-scattering within the aggregate is considered $^{70}$ :

$$
f\left(R_{\mathrm{g}}, \theta, D_{\mathrm{f}}\right)=\left\{\begin{array}{l}
\exp \left[\frac{-\left(q R_{\mathrm{g}}\right)^{2}}{3}\right] \text { if }\left(q R_{\mathrm{g}}\right)^{2}<\frac{3}{2} D_{\mathrm{f}} \\
{\left[\frac{3 D_{\mathrm{f}}}{2 e\left(q R_{\mathrm{g}}\right)^{2}}\right]^{\frac{D_{\mathrm{f}}}{2}} \text { if }\left(q R_{\mathrm{g}}\right)^{2}>\frac{3}{2} D_{\mathrm{f}}}
\end{array}\right.
$$


where it is understood that $q$ is a function of $\theta$ and $\lambda$. For aerosols made of large clusters, only the power-law regime can be considered (second part of Eq. 17). The current expression is in good agreement with the amplitude of the power-law regime proposed by Heinson et al. ${ }^{71}$ but it must be noticed that amplitude may be affected by the aggregate polydispersity ${ }^{44}$. Based on these expressions, the simplest analytical expression for the backscattering cross section can then be found as:

$$
\mathrm{d} C^{\mathrm{bac}}=N_{\mathrm{m}}^{2} \frac{16 \pi^{4} R_{\mathrm{m}}^{6}}{\lambda^{4}} F(m) f^{\mathrm{bac}} C_{\mathrm{p}}
$$

where $F(m)=\left|\left(m^{2}-1\right) /\left(m^{2}+2\right)\right|^{2}, f^{\text {bac }}=f\left(R_{\mathrm{g}}, \theta=\pi, D_{\mathrm{f}}\right)$ and $C_{\mathrm{p}}$ is a correction factor ${ }^{72}$ depending on the width of the aggregate-size distribution. When $C_{\mathrm{p}}=1$, the aggregates are monodisperse, which is the simplest case and the one we apply here. We note that Sorensen and Wang ${ }^{72}$ find that $C_{\mathrm{p}}=1.57$ for diffusion-limited cluster aggregates $(\mathrm{DLCA})$ with $D_{\mathrm{f}}=1.75$. It is not clear what value for $C_{\mathrm{p}}$ applies to real-world $\mathrm{BC}$ aerosols in the atmosphere since their formation likely does not follow pure DLCA or reaction-limited cluster aggregation processes ${ }^{73}$. For this reason, we choose $C_{\mathrm{p}}=1$ and anticipate further refinement of the value form future backscattering measurements from real-world aggregates in the atmosphere.

An analytical expression of the lidar ratio can also be found as:

$$
L R_{\mathrm{bc}}=\frac{\mathrm{d} C^{\mathrm{bac}}}{\mathrm{d} C^{\mathrm{ext}}}=\frac{\lambda^{3}}{2 \pi^{2} N_{\mathrm{m}} R_{\mathrm{m}}^{3} f^{\mathrm{bac}}} \frac{E(m)}{F(m)}+\frac{8 \pi}{3} \frac{g}{f^{\mathrm{bac}}}
$$

which has units of solid angle and where $E(m)=\operatorname{Im}\left\{\left(m^{2}-1\right) /\left(m^{2}+2\right)\right\}$ and $g$ is a correction factor also provided by Dobbins and Megaridis ${ }^{69}$ as:

$$
g=\left[1+\frac{4}{3 D_{\mathrm{f}}}\left(\frac{2 \pi R_{\mathrm{g}}}{\lambda}\right)^{2}\right]^{-\frac{D_{\mathrm{f}}}{2}} .
$$

Next, we summarize the various parameters used to determine $L R_{\mathrm{bc}}$ and $\mathrm{d} C^{\mathrm{bac}}$ for BC particles in the RDG-FA theory. The monomer radius $R_{\mathrm{m}}$ of BC monomers is typically $\sim 5-30 \mathrm{~nm}$, the number of monomers per aggregates $N_{\mathrm{m}}$ ranges between a few tens to a few hundreds, and the fractal dimension, used in the evaluation of $f^{\text {bac }}$ and $g$, is typically $D_{\mathrm{f}}=1.8$. The radius of gyration $R_{\mathrm{g}}$ is a measure of overall aggregate size and can be estimated from the fractal scaling law ${ }^{44}$. All of these parameters can be determined from electron microscopy analysis ${ }^{74}$. The ratio $E(m) / F(m)$ depends on the aggregate's chemical

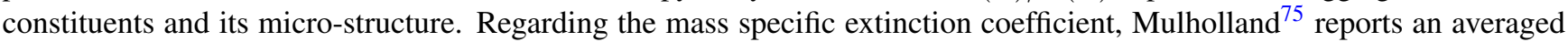
value of $\sigma^{\text {ext }}=8.7 \pm 1.1 \mathrm{~m}^{2} / \mathrm{g}$ which is consistent with the results reported by $\mathrm{Liu}^{4}$ for mature soot or BC aerosols with $0.32 \leq E(m) \leq 0.4$. The refractive index is $m=1.48+i 0.84$ provided by Bescond ${ }^{76}$ is believed to be representative of graphitic soot material representative of $\mathrm{BC}$. Based on this refractive index, $E(\mathrm{~m}) / F(\mathrm{~m})=1.16$ and $F(\mathrm{~m})=0.35$. Thus, the differential backscattering cross-section and lidar ratio are estimated at $\mathrm{d} C_{\mathrm{bac}} / \mathrm{d} \Omega=6.4 \pm 0.6 \times 10^{-4} \mu \mathrm{m}^{2} \mathrm{sr}^{-1}$ and $\mathrm{LR}=125.3 \pm 5.0 \mathrm{sr}$, respectively.

The RDG-FA theory can be derived from the Maxwell equations, which is done in the Appendix of Sorensen et al. ${ }^{77}$ In particular, the derivation highlights important aspects of the various approximations made. First, the assumption that the electromagnetic field is uniform within a given monomer is not strictly true. For a monomer radius of $R_{\mathrm{m}}=30 \mathrm{~nm}$, the largest we consider, the quantity $x_{\mathrm{m}}|m-1|$ used to motivate the RDG-FA treatment evaluates to $\sim 0.34$, and thus, does not necessarily meet the $x_{\mathrm{m}}|m-1| \ll 1$ criterion. Second, the assumption that monomer-to-monomer multiple scattering (within a

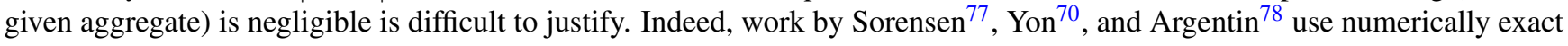
simulations to show that multiple scattering is both present and wavelength dependent, as one would intuitively expect for monomers in physical contact in an aggregate. For example, in relation to the assumption of no monomer-monomer multiple scattering, Lu and Sorensen ${ }^{79}$ find evidence for such scattering in soot aggregates via depolarization measurements. Third, Sorensen et al. ${ }^{77}$ shows that the RDG-FA theory does not satisfy energy conservation when used to calculate an aggregate's extinction cross section via the optical theorem. Such observations present a paradox in that, despite its shortcomings, the RDG-FA does agree well with light-scattering measurements of BC fractal aggregates ${ }^{44}$, including backscattering ${ }^{66}$. The resolution of this paradox is explained by Berg ${ }^{80}$. In short, while the electromagnetic fields within the monomers is indeed not uniform, once the scattering properties of an aggregate are averaged over random orientations, the RDG-FA theory becomes a good approximation due to fortuitous interference cancellations. 


\section{References}

1. Bowman, D. M. J. S. et al. Fire in the earth system. Science 324, 481-484, DOI: $10.1126 /$ science.1163886 (2009). https://science.sciencemag.org/content/324/5926/481.full.pdf.

2. Chýlek, P. et al. Black carbon and absorption of solar radiation by clouds. J. Geophys. Res. Atmospheres 101, 23365-23371, DOI: https://doi.org/10.1029/96JD01901 (1996). https://agupubs.onlinelibrary.wiley.com/doi/pdf/10.1029/96JD01901.

3. Bond, T. C. et al. Bounding the role of black carbon in the climate system: A scientific assessment. J. Geophys. Res. Atmospheres 118, 5380-5552, DOI: https://doi.org/10.1002/jgrd.50171 (2013).

4. Liu, F. et al. Review of recent literature on the light absorption properties of black carbon: Refractive index, mass absorption cross section, and absorption function. Aerosol Sci. Technol. 54, 33-51, DOI: 10.1080/02786826.2019.1676878 (2020). https://doi.org/10.1080/02786826.2019.1676878.

5. Cherian, R., Quaas, J., Salzmann, M. \& Tomassini, L. Black carbon indirect radiative effects in a climate model. Tellus B: Chem. Phys. Meteorol. 69, 1369342, DOI: 10.1080/16000889.2017.1369342 (2017). https://doi.org/10.1080/16000889. 2017.1369342.

6. Jansen, K. L. et al. Associations between health effects and particulate matter and black carbon in subjects with respiratory disease. Environ. health perspectives 113, 1741-1746, DOI: 10.1289/ehp.8153 (2005).

7. Pérez, N. et al. Variability of particle number, black carbon, and pm10, pm2.5, and pm1 levels and speciation: Influence of road traffic emissions on urban air quality. Aerosol Sci. Technol. 44, 487-499, DOI: 10.1080/02786821003758286 (2010).

8. Schumann, U. et al. In situ observations of particles in jet aircraft exhausts and contrails for different sulfur-containing fuels. J. Geophys. Res. Atmospheres 101, 6853-6869, DOI: https://doi.org/10.1029/95JD03405 (1996). https://agupubs. onlinelibrary.wiley.com/doi/pdf/10.1029/95JD03405.

9. Delhaye, D. et al. The mermose project: Characterization of particulate matter emissions of a commercial aircraft engine. J. Aerosol Sci. 105, 48-63, DOI: https://doi.org/10.1016/j.jaerosci.2016.11.018 (2017).

10. Kärcher, B. Formation and radiative forcing of contrail cirrus. Nat. communications 9, 1-17, DOI: 10.1038/ s41467-018-04068-0 (2018).

11. Teoh, R., Schumann, U., Majumdar, A. \& Stettler, M. E. J. Mitigating the climate forcing of aircraft contrails by small-scale diversions and technology adoption. Environ. Sci. \& Technol. 54, 2941-2950, DOI: 10.1021/acs.est.9b05608 (2020). PMID: 32048502.

12. Ramanathan, V. \& Carmichael, G. Global and regional climate changes due to black carbon. Nat Geosci 1, DOI: 10.1038/ngeo156 (2008).

13. Cooke, W. F. \& Wilson, J. J. N. A global black carbon aerosol model. J. Geophys. Res. Atmospheres 101, 19395-19409, DOI: https://doi.org/10.1029/96JD00671 (1996). https://agupubs.onlinelibrary.wiley.com/doi/pdf/10.1029/96JD00671.

14. Bond, T. C., Anderson, T. L. \& Campbell, D. Calibration and intercomparison of filter-based measurements of visible light absorption by aerosols. Aerosol Sci. Technol. 30, 582-600, DOI: 10.1080/027868299304435 (1999).

15. Ajtai, T. et al. A novel multi- wavelength photoacoustic spectrometer for the measurement of the uv-vis-nir spectral absorption coefficient of atmospheric aerosols. J. aerosol science 41, 1020-1029, DOI: 10.1016/j.jaerosci.2010.07.008 (2010).

16. Sedlacek, A. \& Lee, J. Photothermal interferometric aerosol absorption spectrometry. Aerosol Sci. Technol. 41, 1089-1101, DOI: $10.1080 / 02786820701697812$ (2007).

17. Kim, S.-W., Cho, C. \& Rupakheti, M. Estimating contributions of black and brown carbon to solar absorption from aethalometer and aeronet measurements in the highly polluted kathmandu valley, nepal. Atmospheric Res. 247, 105164, DOI: 10.1016/j.atmosres.2020.105164 (2021).

18. Moosmüller, H., Chakrabarty, R. \& Arnott, W. Aerosol light absorption and its measurement: A review. J. Quant. Spectrosc. Radiat. Transf. 110, 844-878, DOI: 0.1016/j.jqsrt.2009.02.035 (2009).

19. Stephens, M., Turner, N. \& Sandberg, J. Particle identification by laser-induced incandescence in a solid-state laser cavity. Appl. Opt. 42, 3726-3736, DOI: 10.1364/AO.42.003726 (2003).

20. Schwarz, J. P. et al. The detection efficiency of the single particle soot photometer. Aerosol Sci. Technol. 44, 612-628, DOI: 10.1080/02786826.2010.481298 (2010). https://doi.org/10.1080/02786826.2010.481298.

21. Onasch, T. B. et al. Soot particle aerosol mass spectrometer: Development, validation, and initial application. Aerosol Sci. Technol. 46, 804-817, DOI: 10.1080/02786826.2012.663948 (2012). 
22. Zhao, W. et al. Determination of equivalent black carbon mass concentration from aerosol light absorption using variable mass absorption cross section. Atmospheric Meas. Tech. 14, 1319-1331, DOI: 10.5194/amt-14-1319-2021 (2021).

23. Zuev, V. \& Wood, J. Laser Beams in the Atmosphere (Consultants Bureau, 1982).

24. Measures, R. M. Laser remote sensing: Fundamentals and applications. New York, Wiley-Interscience (1984).

25. Bissonnette, L. R. Lidar and Multiple Scattering, 43-103 (Springer New York, New York, NY, 2005).

26. Ceolato, R. \& Berg, M. J. Aerosol light extinction and backscattering: A review with a lidar perspective. J. Quant. Spectrosc. Radiat. Transf. 262, 107492, DOI: https://doi.org/10.1016/j.jqsrt.2020.107492 (2021).

27. Althausen, D. et al. Portable raman lidar polly xt for automated profiling of aerosol backscatter, extinction, and depolarization. J. Atmospheric Ocean. Technol. 26, 2366-2378, DOI: 10.1175/2009JTECHA1304.1 (2009).

28. Mazzoleni, C., Kuhns, H. D. \& Moosmüller, H. Monitoring automotive particulate matter emissions with lidar: A review. Remote. Sens. 2, 1077-1119, DOI: 10.3390/rs2041077 (2010).

29. Edner, H., Ragnarson, P. \& Wallinder, E. Industrial emission control using lidar techniques. Environ. Sci. \& Technol. 29, 330-337, DOI: 10.1021/es00002a008 (1995).

30. Schröter, M., Obermeier, A., Brüggemann, D., Plechschmidt, M. \& Klemm, O. Remote monitoring of air pollutant emissions from point sources by a mobile lidar/sodar system. J. Air \& Waste Manag. Assoc. 53, 716-723, DOI: 10.1080/ 10473289.2003.10466213 (2003).

31. Evgenieva, T. T. et al. Lidar and spectroradiometer measurements of atmospheric aerosol optical characteristics over an urban area in sofia, bulgaria. Int. J. Remote. Sens. 30, 6381-6401, DOI: 10.1080/01431160902865764 (2009).

32. Brown, D. M., Thrush, E. \& Thomas, M. E. Chamber lidar measurements of biological aerosols. Appl Opt 50, 717-24, DOI: 10.1364/AO.50.000717 (2011).

33. Guerrero-Rascado, J. L. et al. Multispectral elastic scanning lidar for industrial flare research: characterizing the electronic subsystem and application. Opt. Express 22, 31063-31077, DOI: 10.1364/OE.22.031063 (2014).

34. Brown, D. M. et al. Lidar measurements of solid rocket propellant fire particle plumes. Appl Opt 55, 4657-69, DOI: 10.1364/AO.55.004657 (2016).

35. Ong, P. M., Lagrosas, N., Shiina, T. \& Kuze, H. Surface aerosol properties studied using a near-horizontal lidar. Atmosphere 11, DOI: 10.3390/atmos11010036 (2019).

36. Koch, D. et al. Evaluation of black carbon estimations in global aerosol models. Atmospheric Chem. Phys. 9, 9001-9026, DOI: 10.5194/acp-9-9001-2009 (2009).

37. Vignati, E. et al. Sources of uncertainties in modelling black carbon at the global scale. Atmospheric Chem. Phys. 10, 2595-2611, DOI: 10.5194/acp-10-2595-2010 (2010).

38. Andersson, C. et al. Match-salsa - multi-scale atmospheric transport and chemistry model coupled to the salsa aerosol microphysics model - part 1: Model description and evaluation. Geosci. Model. Dev. 8, 171-189, DOI: 10.5194/ gmd-8-171-2015 (2015).

39. Kort, A. et al. Quantification of soot deposit on a resistive sensor: Proposal of an experimental calibration protocol. $J$. Aerosol Sci. 156, 105783, DOI: 10.1016/j.jaerosci.2021.105783 (2021).

40. Liu, N., Lei, J., Gao, W., Chen, H. \& Xie, X. Combustion dynamics of large-scale wildfires. Proc. Combust. Inst. DOI: 10.1016/j.proci.2020.11.006 (2021).

41. Barfknecht, T. R. Toxicology of soot. Prog. Energy Combust. Sci. 9, 199-237, DOI: https://doi.org/10.1016/0360-1285(83) 90002-3 (1983).

42. Bové, H. et al. Ambient black carbon particles reach the fetal side of human placenta. Nat. Commun. 10, DOI: 10.1038/s41467-019-11654-3 (2019).

43. Treacy, M. M. J. \& Rice, S. B. Catalyst particle sizes from rutherford scattered intensities. J. Microsc. 156, 211-234, DOI: https://doi.org/10.1111/j.1365-2818.1989.tb02920.x (1989). https://onlinelibrary.wiley.com/doi/pdf/10.1111/j.1365-2818. 1989.tb02920.x.

44. Sorensen, C. M. Light scattering by fractal aggregates: A review. Aerosol Sci. Technol. 35, 648-687, DOI: 10.1080/ 02786820117868 (2001).

45. Apicella, B. et al. Soot nanostructure evolution in premixed flames by high resolution electron transmission microscopy (hrtem). Proc. Combust. Inst. 35, 1895-1902, DOI: https://doi.org/10.1016/j.proci.2014.06.121 (2015). 
46. Jurkiewicz, K., Pawlyta, M. \& Burian, A. Structure of carbon materials explored by local transmission electron microscopy and global powder diffraction probes. $C$ 4, DOI: $10.3390 / c 4040068$ (2018).

47. Singh, M. \& Vander Wal, R. L. Nanostructure quantification of carbon blacks. C 5, DOI: $10.3390 /$ c5010002 (2019).

48. Apicella, B. et al. Hrtem and eels investigations of flame-formed soot nanostructure. Fuel 225, 218-224, DOI: https: //doi.org/10.1016/j.fuel.2018.03.091 (2018).

49. Leapman, R. D., Fejes, P. L. \& Silcox, J. Orientation dependence of core edges from anisotropic materials determined by inelastic scattering of fast electrons. Phys. Rev. B 28, 2361-2373, DOI: 10.1103/PhysRevB.28.2361 (1983).

50. Papworth, A. J., Kiely, C. J., Burden, A. P., Silva, S. R. P. \& Amaratunga, G. A. J. Electron-energy-loss spectroscopy characterization of the $s p^{2}$ bonding fraction within carbon thin films. Phys. Rev. B 62, 12628-12631, DOI: 10.1103/ PhysRevB.62.12628 (2000).

51. Ceolato, R., Bedoya-Velásquez, A. E. \& Mouysset, V. Short-range elastic backscatter micro-lidar for quantitative aerosol profiling with high range and temporal resolution. Remote. Sens. 12, DOI: 10.3390/rs12203286 (2020).

52. Bedoya-Velásquez, A. et al. Ceilometer inversion method using water-vapor correction from co-located microwave radiometer for aerosol retrievals. Atmospheric Res. 250, 105379, DOI: https://doi.org/10.1016/j.atmosres.2020.105379 (2021).

53. Choi, Y., Ghim, Y. S., Zhang, Y., Park, S.-M. \& Song, I.-h. Estimation of surface concentrations of black carbon from long-term measurements at aeronet sites over korea. Remote. Sens. 12, DOI: 10.3390/rs12233904 (2020).

54. Vaughan, M. et al. Calipso lidar calibration at $1064 \mathrm{~nm}$ : version 4 algorithm. Atmospheric Meas. Tech. 12, 51-82, DOI: 10.5194/amt-12-51-2019 (2019).

55. Lopatin, A. et al. Enhancement of aerosol characterization using synergy of lidar and sun-photometer coincident observations: the garrlic algorithm. Atmospheric Meas. Tech. 6, 2065-2088, DOI: 10.5194/amt-6-2065-2013 (2013).

56. Benavent-Oltra, J. A. et al. Different strategies to retrieve aerosol properties at night-time with the grasp algorithm. Atmospheric Chem. Phys. 19, 14149-14171, DOI: 10.5194/acp-19-14149-2019 (2019).

57. Mishchenko, M. I. et al. First-principles modeling of electromagnetic scattering by discrete and discretely heterogeneous random media. Phys. Reports 632, 1 - 75, DOI: https://doi.org/10.1016/j.physrep.2016.04.002 (2016). First-principles modeling of electromagnetic scattering by discrete and discretely heterogeneous random media.

58. Fernald, F. G., Herman, B. M. \& Reagan, J. A. Determination of aerosol height distributions by lidar. J. Appl. meteorology 11, 482-489, DOI: 10.1175/1520-0450(1972)011<0482:DOAHDB>2.0.CO;2 (1972).

59. Klett, J. D. Stable analytical inversion solution for processing lidar returns. Appl. optics 20, 211-220, DOI: 10.1364/AO. 20.000211 (1981).

60. Fernald, F. G. Analysis of atmospheric lidar observations: some comments. Appl. optics 23, 652-653, DOI: 10.1364/AO. 23.000652 (1984).

61. Kovalev, V. A. \& Moosmüller, H. Distortion of particulate extinction profiles measured with lidar in a two-component atmosphere. Appl. optics 33, 6499-6507, DOI: 10.1364/AO.33.006499 (1994).

62. Comerón, A., Muñoz-Porcar, C., Rocadenbosch, F., Rodríguez-Gómez, A. \& Sicard, M. Current research in lidar technology used for the remote sensing of atmospheric aerosols. Sensors 17, DOI: 10.3390/s17061450 (2017).

63. Lagrosas, N. et al. Correlation study between suspended particulate matter and portable automated lidar data. J. Aerosol Sci. 36, 439-454, DOI: https://doi.org/10.1016/j.jaerosci.2004.10.007 (2005).

64. Yurkin, M. \& Hoekstra, A. The discrete dipole approximation: An overview and recent developments. J. Quant. Spectrosc. Radiat. Transf. 106, 558-589, DOI: https://doi.org/10.1016/j.jqsrt.2007.01.034 (2007). IX Conference on Electromagnetic and Light Scattering by Non-Spherical Particles.

65. Köylü, Ü. Ö. \& Faeth, G. M. Optical Properties of Soot in Buoyant Laminar Diffusion Flames. J Heat Transf 116, 971-979, DOI: 10.1115/1.2911473 (1994).

66. Ceolato, R., Paulien, L., Maughan, J. B., Sorensen, C. M. \& Berg, M. J. Radiative properties of soot fractal superaggregates including backscattering and depolarization. J. Quant. Spectrosc. Radiat. Transf. 247, 106940, DOI: https://doi.org/10. 1016/j.jqsrt.2020.106940 (2020).

67. Wang, G. \& Sorensen, C. M. Experimental test of the rayleigh-debye-gans theory for light scattering by fractal aggregates. Appl. Opt. 41, 4645-4651, DOI: 10.1364/AO.41.004645 (2002). 
68. Yon, J., Morán, J., Ouf, F.-X., Mazur, M. \& Mitchell, J. From monomers to agglomerates: A generalized model for characterizing the morphology of fractal-like clusters. J. Aerosol Sci. 151, 105628, DOI: 10.1016/j.jaerosci.2020.105628 (2021).

69. Dobbins, R. A. \& Megaridis, C. M. Absorption and scattering of light by polydisperse aggregates. Appl. optics 30, 4747-4754, DOI: 10.1364/AO.30.004747 (1991).

70. Yon, J. et al. Effects of multiple scattering on radiative properties of soot fractal aggregates. J. Quant. Spectrosc. Radiat. Transf. 133, 374-381, DOI: https://doi.org/10.1016/j.jqsrt.2013.08.022 (2014).

71. Heinson, W., Sorensen, C. \& Chakrabarti, A. A three parameter description of the structure of diffusion limited cluster fractal aggregates. J. Colloid Interface Sci. 375, 65-69, DOI: https://doi.org/10.1016/j.jcis.2012.01.062 (2012).

72. Sorensen, C. M. \& Wang, G. M. Size distribution effect on the power law regime of the structure factor of fractal aggregates. Phys. Rev. E 60, 7143-7148, DOI: 10.1103/PhysRevE.60.7143 (1999).

73. China, S., Salvadori, N. \& Mazzoleni, C. Effect of traffic and driving characteristics on morphology of atmospheric soot particles at freeway on-ramps. Environ. Sci. \& Technol. 48, 3128-3135, DOI: 10.1021/es405178n (2014). PMID: 24559238.

74. Ouf, F. X., Yon, J., Ausset, P., Coppalle, A. \& Maillé, M. Influence of sampling and storage protocol on fractal morphology of soot studied by transmission electron microscopy. Aerosol Sci. Technol. 44, 1005-1017, DOI: 10.1080/02786826.2010. 507228 (2010). https://doi.org/10.1080/02786826.2010.507228.

75. Mulholland, G. W. \& Croarkin, C. Specific extinction coefficient of flame generated smoke. Fire Mater. 24, 227-230, DOI: https://doi.org/10.1002/1099-1018(200009/10)24:5<227::AID-FAM742>3.0.CO;2-9 (2000).

76. Bescond, A. et al. Soot optical properties determined by analyzing extinction spectra in the visible near-uv: Toward an optical speciation according to constituents and structure. J. Aerosol Sci. 101, 118-132, DOI: https://doi.org/10.1016/j. jaerosci.2016.08.001 (2016).

77. Sorensen, C. M. et al. Light scattering and absorption by fractal aggregates including soot. J. Quant. Spectrosc. Radiat. Transf. 217, 459-473, DOI: 10.1016/j.jqsrt.2018.05.016 (2018).

78. Argentin, C., Berg, M. J., Mazur, M., Ceolato, R. \& Yon, J. Assessing the limits of rayleigh-debye-gans theory: Phasor analysis of a bisphere. J. Quant. Spectrosc. Radiat. Transf. 264, 107550, DOI: 10.1016/j.jqsrt.2021.107550 (2021).

79. Lu, N. \& Sorensen, C. M. Depolarized light scattering from fractal soot aggregates. Phys. Rev. E 50, 3109-3115 (1994).

80. Berg, M. J. \& Sorensen, C. M. Internal fields of soot fractal aggregates. J. Opt. Soc. Am. A 30, 1947-1954, DOI: 10.1364/JOSAA.30.001947 (2013).

\section{Acknowledgements}

This work was financially supported by ONERA within the framework of the PROMETE project 2017-2021. MB acknowledges support from the National Science Foundation (1665456), the Air Force Office of Scientific Research (FA9550-19-1- 0078), and the Army Research Office (W911NF2120053). The authors are grateful for advice from Nicolas Riviere, Valérie Rialland, Thierry Huet, Charly Mandal, Gérald Lemineur, Gilles Roy, Grégoire Tremblay, and William Heinson. The authors thank Guillaume Huss and Paul-Henri Pioger from Leukos for developing and proving the laser source. ONERA authors acknowledge the General Scientific Directorate (DSG), including Riad Haidar and Laurent Jacquin, for their continued support.

\section{Author contributions statement}

RC conceived the PSR-EBL lidar instrument and supervised the project; RC and AB conducted the experiments; RC, AB, VM and SL developed the processing code and the inverse method; JY, LP, RC, CS, and MB developed the RDG-FA model. All authors analysed the results, read, reviewed and approved the final version of the manuscript.

\section{Additional information}

Accession codes Measured data supporting the findings of this study and the experimental results shown in Fig. 1, Fig. 2, and Fig. 3 are available from the authors upon request. Competing interests The authors declare no competing financial interests. 


\section{Supplementary Files}

This is a list of supplementary files associated with this preprint. Click to download.

- SINC.pdf 